\title{
Natural Elements of Center of Generalized Quantum Groups
}

\author{
Punita Batra and Hiroyuki Yamane
}

\begin{abstract}
This paper gives elements in the (skew) center of the generalized quantum group corresponding to its irreducible finite dimensional modules. Finally we give a conjecture stating that those must form a basis of the center.
\end{abstract}

\section{Introduction}

This paper is a continuation of [4], [5]. Let $V$ be a finite-dimensional real linear space. Let $l:=\operatorname{dim}_{\mathbb{R}} V$, and assume $l \geq 1$. Let $\left\{\alpha_{1}, \ldots, \alpha_{l}\right\}$ be an $\mathbb{R}$-basis of $V$. So $V=\oplus_{i=1}^{l} \mathbb{R} \alpha_{i}$. Let $V_{\mathbb{Z}}:=\oplus_{i=1}^{l} \mathbb{Z} \alpha_{i}$. Then $V_{\mathbb{Z}}$ is a free $\mathbb{Z}$-module with $\operatorname{rank}_{\mathbb{Z}} V_{\mathbb{Z}}=l$. Let $\mathbb{K}$ be a field. Let $\mathbb{K}^{\times}:=\mathbb{K} \backslash\{0\}$. Let $\chi: V_{\mathbb{Z}} \times V_{\mathbb{Z}} \rightarrow \mathbb{K}^{\times}$be a map such that $\chi(\lambda+\mu, \nu)=\chi(\lambda, \nu) \chi(\mu, \nu)$ and $\chi(\lambda, \mu+\nu)=\chi(\lambda, \mu) \chi(\lambda, \nu)$. For the $\chi$, in the same way as the Lusztig way [17, 3.1.1] to define the quantum groups $U_{q}(\mathfrak{g})$, we can define the Hopf algebra $U=U(\chi, \pi)$ over $\mathbb{K}$, which we call the generalized quantum group. ( $U$ is the one of Subsection 2.2 with assuming $V_{\mathbb{Z}}$ to be $\mathfrak{A}$. In Introduction, we may also assume $V_{\mathbb{Z}}=\mathfrak{A}=\mathfrak{A}_{\pi}$. The notation $\pi$ means the map from $\{1, \ldots, l\}$ to $\mathfrak{A}$ defined by $\pi(i):=\alpha_{i}$.) For some $\chi, U$ can be the quantum groups, the small quantum groups at root of 1 , the quantum superalgebras, or the one associated with the Nichols algebras in the Heckenberger's list [10]. Further studies concerning $U$ have been achieved by [1], 2], 3], [6], [11, [12, [13], etc. Let $U=U^{-} \otimes U^{0} \otimes U^{+}$be the triangular decomposition (see (U5)). Let $U=\bigoplus_{\lambda \in V_{\mathbb{Z}}} U_{\lambda}$

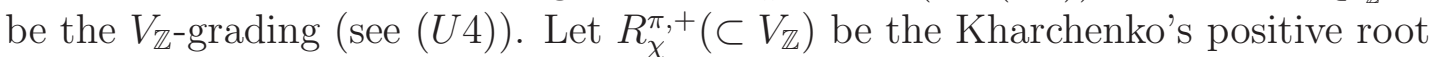
system defined for $U$ (see Theorem 2.2, which was originally given by [15]). As in the assumption of Theorem 2.4, assume that $R_{\chi}^{\pi,+}$ is a finite set and assume that $\chi(\alpha, \alpha) \neq 1$ for all $\alpha \in R_{\chi}^{\pi,+}$. Let $\omega: V_{\mathbb{Z}} \rightarrow \mathbb{K}^{\times}$be the map such that $\omega(\lambda+\mu)=$

THIS VERSION IS ON 17/MAY/2019.

2000 Mathematics Subject Classification: Primary 17B37, 17B10; Secondary 81R50.

HY is partially supported by JSPS Grand-in-Aid for Scientific Research (C), 16K05095. 
$\omega(\lambda) \omega(\mu)$. Let $\mathfrak{Z}_{\omega}(\chi, \pi):=\left\{Z \in U_{0} \mid \forall \lambda \in V_{\mathbb{Z}}, \forall X \in U_{\lambda}, Z X=\omega(\lambda) X Z\right\}$ (see (2.9)). In [5, Theorem 10.4] (see Theorem 2.4), we have the Harish-Chandra type isomorphism $\mathfrak{H C}_{\omega}^{\mathfrak{c}, \pi}: \mathfrak{Z}_{\omega}(\chi, \pi) \rightarrow \mathfrak{B}_{\omega}^{\chi, \pi}$, where $\mathfrak{B}_{\omega}^{\chi, \pi}$ is the $\mathbb{K}$-subspace of $U^{0}$ defined by the equrations $(e 1)_{\beta^{-}}(e 4)_{\beta}$ for all $\beta \in R_{\chi}^{\pi,+}$. As in (3.11), for $\lambda, \mu \in$ $V_{\mathbb{Z}}$, define the $\mathbb{K}$-algebra homomorphism $\Lambda_{\lambda, \mu ; \omega}^{\chi}: U^{0} \rightarrow \mathbb{K}$ by $\Lambda_{\lambda, \mu ; \omega}^{\chi}\left(K_{\lambda^{\prime}} L_{\mu^{\prime}}\right):=$ $\chi\left(\lambda, \mu^{\prime}\right) \chi\left(\lambda^{\prime}, \mu\right) \omega\left(\lambda^{\prime}\right)$ for $\lambda^{\prime}, \mu^{\prime} \in V_{\mathbb{Z}}$, where $\left\{K_{\lambda^{\prime}} L_{\mu^{\prime}} \mid \lambda^{\prime}, \mu^{\prime} \in V_{\mathbb{Z}}\right\}$ is a $\mathbb{K}$-basis of $U^{0}$ (see $(U 3))$. As in (3.12), let $\operatorname{Fin}_{\omega}^{\chi}:=\left\{(\lambda, \mu) \in V_{\mathbb{Z}} \times V_{\mathbb{Z}} \mid \operatorname{dim} \mathcal{L}\left(\Lambda_{\lambda, \mu ; \omega}^{\chi}\right)<\infty\right\}$, where $\mathcal{L}\left(\Lambda_{\lambda, \mu ; \omega}^{\chi}\right)$ is the finite-dimensional simple $U$-modules defined in the way that $\Lambda_{\lambda, \mu ; \omega}^{\chi}$ is regarded as its highest weight. By our main result Theorem 3.7, for each $(\lambda, \mu) \in \operatorname{Fin}_{\omega}^{\chi}$, we have $Z_{\lambda, \mu ; \omega}^{\chi} \in \mathfrak{Z}_{\omega}(\chi, \pi)$ so that $\mathfrak{H C}_{\omega}^{\chi, \pi}\left(Z_{\lambda, \mu ; \omega}^{\chi}\right)$ can be viewed as the character of $\mathcal{L}\left(\Lambda_{\lambda, \mu ; \omega}^{\chi}\right)$. As a final stage of this paper, we state Conjecture (3.13), which states that $\left\{Z_{\lambda, \mu ; \omega}^{\chi} \mid(\lambda, \mu) \in \operatorname{Fin}_{\omega}^{\chi}\right\}$ is a $\mathbb{K}$-basis of $\mathfrak{Z}_{\omega}(\chi, \pi)$.

\section{Generalized quantum groups}

\subsection{Preliminary}

For $x, y \in \mathbb{R}$, let $J_{x, y}:=\{z \in \mathbb{Z} \mid x \leq z \leq y\}$. Let $\mathbb{K}$ be a field. Let $\mathbb{K}^{\times}:=$ $\mathbb{K} \backslash\{0\}$. For $n \in \mathbb{Z}_{\geq 0}$ and $x \in \mathbb{K}$, let $(n)_{x}:=\sum_{r=1}^{n} x^{r-1}$, and $(n)_{x} !:=\prod_{r=1}^{n}(r)_{x}$. For $n \in \mathbb{Z}_{\geq 0}, m \in J_{0, n}$ and $x \in \mathbb{K}$, define $\left(\begin{array}{l}r \\ m\end{array}\right)_{x} \in \mathbb{K}$ by $\left(\begin{array}{l}n \\ 0\end{array}\right)_{x}:=\left(\begin{array}{l}n \\ n\end{array}\right)_{x}:=1$, and $\left(\begin{array}{c}n \\ m\end{array}\right)_{x}:=\left(\begin{array}{c}n-1 \\ m\end{array}\right)_{x}+x^{n-m}\left(\begin{array}{c}n-1 \\ m-1\end{array}\right)_{x}=x^{m}\left(\begin{array}{c}n-1 \\ m\end{array}\right)_{x}+\left(\begin{array}{c}n-1 \\ m-1\end{array}\right)_{x}$ (if $\left.m \in J_{1, n-1}\right)$. If $(m)_{x} !(n-m)_{x} ! \neq 0$, then $\left(\begin{array}{l}n \\ m\end{array}\right)_{x}=\frac{(n)_{x} !}{(m)_{x} !(n-m)_{x} !}$. For $x, y, z \in \mathbb{K}$, and $n \in \mathbb{N}$, we have $\prod_{t=0}^{n-1}\left(y+x^{t} z\right)=\sum_{m=0}^{n} x^{\frac{m(m-1)}{2}}\left(\begin{array}{c}n \\ m\end{array}\right)_{x} y^{n-m} z^{m}$.

For $x \in \mathbb{K}^{\times}$, define $\hat{o}(x) \in \mathbb{Z}_{\geq 0} \backslash\{1\}$ by

$$
\hat{o}(x):=\left\{\begin{array}{l}
\min \left\{r^{\prime} \in J_{2, \infty} \mid\left(r^{\prime}\right)_{x} !=0\right\} \text { if }\left(r^{\prime \prime}\right)_{x} !=0 \text { for some } r^{\prime \prime} \in J_{2, \infty}, \\
0 \text { otherwise. }
\end{array}\right.
$$

Let $\mathfrak{A}$ be an abelian group. Then $\mathfrak{A}$ is a $\mathbb{Z}$-module. Let $\chi: \mathfrak{A} \times \mathfrak{A} \rightarrow \mathbb{K}^{\times}$be a map such that

$$
\forall \lambda, \forall \mu, \forall \nu \in \mathfrak{A}, \chi(\lambda+\mu, \nu)=\chi(\lambda, \nu) \chi(\mu, \nu), \chi(\lambda, \mu+\nu)=\chi(\lambda, \mu) \chi(\lambda, \nu) .
$$

Let $l \in \mathbb{N}$ and $I:=J_{1, l}$. Assume that there exists an injection $\pi: I \rightarrow \mathfrak{A}$ such that $\pi(I)$ is a $\mathbb{Z}$-basis of $\operatorname{Span}_{\mathbb{Z}}(\pi(I))$. Let $\mathfrak{A}_{\pi}:=\operatorname{Span}_{\mathbb{Z}}(\pi(I))$. Let $\alpha_{i}:=\pi(i)$ $(i \in I)$. Then $\mathfrak{A}_{\pi}:=\oplus_{i \in I} \mathbb{Z} \alpha_{i}$. Let $\mathfrak{A}_{\pi}^{+}:=\oplus_{i \in I} \mathbb{Z}_{\geq 0} \alpha_{i}$.

\subsection{Definition of $U=U(\chi, \pi)$}

The facts mentioned in this subsection is well-known and can be proved in a standard way introduced by Drinfeld [7] and Lusztig [17, 3.1.1].

There exists a unique associative $\mathbb{K}$-algebra (with 1$) U=U(\chi, \pi)$ satisfying the following conditions $(U 1)-(U 6)$. 
$(U 1)$ As a $\mathbb{K}$-algebra, $U$ is generated by the elements:

$$
K_{\lambda}, L_{\lambda}(\lambda \in \mathfrak{A}), \quad E_{i}, F_{i}(i \in I) .
$$

(U2) The elements of (2.3) satisfy the following relations.

$$
\begin{aligned}
& K_{0}=L_{0}=1, K_{\lambda} K_{\mu}=K_{\lambda+\mu}, L_{\lambda} L_{\mu}=L_{\lambda+\mu}, K_{\lambda} L_{\mu}=L_{\mu} K_{\lambda}, \\
& K_{\lambda} E_{i}=\chi\left(\lambda, \alpha_{i}\right) E_{i} K_{\lambda}, L_{\lambda} E_{i}=\chi\left(-\alpha_{i}, \lambda\right) E_{i} L_{\lambda}, \\
& K_{\lambda} F_{i}=\chi\left(\lambda,-\alpha_{i}\right) F_{i} K_{\lambda}, L_{\lambda} F_{i}=\chi\left(\alpha_{i}, \lambda\right) F_{i} L_{\lambda}, \\
& {\left[E_{i}, F_{j}\right]=\delta_{i j}\left(-K_{\alpha_{i}}+L_{\alpha_{i}}\right) .}
\end{aligned}
$$

(U3) Define the map $\varsigma_{1}: \mathfrak{A} \times \mathfrak{A} \rightarrow U$ by $\varsigma_{1}(\lambda, \mu):=K_{\lambda} L_{\mu}$. Define the $\mathbb{K}$ subalgebra $U^{0}=U^{0}(\chi, \pi)$ of $U$ by $U^{0}:=\operatorname{Span}_{\mathbb{K}}\left(\varsigma_{1}(\mathfrak{A} \times \mathfrak{A})\right)$. Then $\varsigma_{1}$ is injective, and $\varsigma_{1}(\mathfrak{A} \times \mathfrak{A})$ is a $\mathbb{K}$-basis of $U^{0}$.

(U4) There exist $\mathbb{K}$-subspaces $U_{\lambda}=U(\chi, \pi)_{\lambda}$ of $U$ for $\lambda \in \mathfrak{A}_{\pi}$ satisfying the following conditions $(U 4-1)-(U 4-3)$.

$(U 4-1)$ We have $U^{0} \subset U_{0}$ and $E_{i} \in U_{\alpha_{i}}, F_{i} \in U_{-\alpha_{i}}(i \in I)$.

$(U 4-2)$ We have $U_{\lambda} U_{\mu} \subset U_{\lambda+\mu}\left(\lambda, \mu \in \mathfrak{A}_{\pi}\right)$.

$(U 4-3)$ We have $U=\oplus_{\lambda \in \mathfrak{A}_{\pi}} U_{\lambda}$ as a $\mathbb{K}$-linear spaces.

$(U 5)$ Let $U^{+}=U^{+}(\chi, \pi)$ (resp. $\left.U^{-}=U^{-}(\chi, \pi)\right)$ be the $\mathbb{K}$-subalgebra (with 1 ) of $U$ generated by $E_{i}$ (resp. $\left.F_{i}\right)(i \in I)$. Define the $\mathbb{K}$-linear homomorphism $\varsigma_{2}: U^{-} \otimes_{\mathbb{K}} U^{0} \otimes_{\mathbb{K}} U^{+} \rightarrow U(\chi, \pi)$ by $\varsigma_{2}(Y \otimes Z \otimes X):=Y Z X$. Then $\varsigma_{2}$ is a $\mathbb{K}$-linear isomorphism.

(U6) For $\lambda \in \mathfrak{A}_{\pi}$, define $U_{\lambda}^{+}=U^{+}(\chi, \pi)_{\lambda}$ (resp. $\left.U_{\lambda}^{-}=U^{-}(\chi, \pi)_{\lambda}\right)$ by $U_{\lambda}^{+}:=$ $U^{+} \cap U_{\lambda}$ (resp. $U_{\lambda}^{-}=U^{-} \cap U_{\lambda}$ ). Then for $\lambda \in \mathfrak{A}_{\pi}^{+} \backslash\{0\}$, we have $\left\{X \in U_{\lambda}^{+} \mid \forall i \in\right.$ $\left.I,\left[X, F_{i}\right]=0\right\}=\{0\}$ and $\left\{Y \in U_{-\lambda}^{-} \mid \forall i \in I,\left[E_{i}, Y\right]=0\right\}=\{0\}$.

Notice that $U^{+}=\oplus_{\lambda \in \mathfrak{A}_{\pi}^{+}} U_{\lambda}^{+}, U^{-}=\oplus_{\lambda \in \mathfrak{A}_{\pi}^{+}} U_{-\lambda}^{-}, U_{0}^{+}=U_{0}^{-}=\mathbb{K} \cdot 1_{U}$ and $U_{\alpha_{i}}^{+}=$ $\mathbb{K} \cdot E_{i}, U_{-\alpha_{i}}^{-}=\mathbb{K} \cdot F_{i}(i \stackrel{\in}{\in})$.

We also regard $U=U(\chi, \pi)$ as a Hopf algebra $(U, \Delta, S, \varepsilon)$ by

$$
\begin{aligned}
& \Delta\left(K_{\lambda}\right)=K_{\lambda} \otimes K_{\lambda}, \Delta\left(L_{\lambda}\right)=L_{\lambda} \otimes L_{\lambda}, \Delta\left(E_{i}\right)=E_{i} \otimes 1+K_{\alpha_{i}} \otimes E_{i}, \\
& \Delta\left(F_{i}\right)=F_{i} \otimes L_{\alpha_{i}}+1 \otimes F_{i}, S\left(K_{\lambda}\right)=K_{-\lambda}, S\left(L_{\lambda}\right)=L_{-\lambda}, \\
& S\left(E_{i}\right)=-K_{-\alpha_{i}} E_{i}, S\left(F_{i}\right)=-F_{i} L_{-\alpha_{i}}, \\
& \varepsilon\left(K_{\lambda}\right)=\varepsilon\left(L_{\lambda}\right)=1, \varepsilon\left(E_{i}\right)=\varepsilon\left(F_{i}\right)=0 .
\end{aligned}
$$

Let $U^{+, b}:=\oplus_{\lambda \in \mathfrak{A}} U^{+} K_{\lambda}$, and $U^{-, b}:=\oplus_{\lambda \in \mathfrak{A}} U^{-} L_{\lambda}$. Then $U=\operatorname{Span}_{\mathbb{K}}\left(U^{-, b} U^{+, b}\right)=$ $\operatorname{Span}_{\mathbb{K}}\left(U^{+, b} U^{-, b}\right)$. 
As in a standard way (see [7]), we have a bilinear form $\vartheta=\vartheta^{\chi, \pi}: U^{+, b} \times U^{-, b} \rightarrow$ $\mathbb{K}$ having the following properties, see also Subsection 3.1 below.

$$
\begin{aligned}
& \vartheta\left(K_{\lambda}, L_{\mu}\right)=\chi(\lambda, \mu), \vartheta\left(E_{i}, F_{j}\right)=\delta_{i j}, \vartheta\left(K_{\lambda}, F_{j}\right)=\vartheta\left(E_{i}, L_{\lambda}\right)=0, \\
& \vartheta\left(X^{+} Y^{+}, X^{-}\right)=\sum_{k^{-}} \vartheta\left(X^{+},\left(X^{-}\right)_{k^{-}}^{(2)}\right) \vartheta\left(Y^{+},\left(X^{-}\right)_{k^{-}}^{(1)}\right), \\
& \vartheta\left(X^{+}, X^{-} Y^{-}\right)=\sum_{k^{+}} \vartheta\left(\left(X^{+}\right)_{k^{+}}^{(1)}, X^{-}\right) \vartheta\left(\left(X^{+}\right)_{k^{+}}^{(2)}, Y^{-}\right), \\
& \vartheta\left(S\left(X^{+}\right), X^{-}\right)=\vartheta\left(X^{+}, S^{-1}\left(\tilde{X}^{-}\right)\right), \\
& \vartheta\left(X^{+}, 1\right)=\varepsilon\left(X^{+}\right), \vartheta\left(1, X^{-}\right)=\varepsilon\left(X^{-}\right), \\
& X^{-} X^{+}=\sum_{r^{+}, r^{-}} \vartheta\left(\left(X^{+}\right)_{r^{+}}^{\prime,(1)}, S\left(\left(X^{-}\right)_{r^{-}}^{\prime,(1)}\right)\right) \vartheta\left(\left(X^{+}\right)_{r^{+}}^{\prime,(3)},\left(X^{-}\right)_{r^{-}}^{\prime,(3)}\right) \\
& \cdot\left(X^{+}\right)_{r^{+}}^{\prime,(2)}\left(X^{-}\right)_{r^{-}}^{\prime,(2)}, \\
& X^{+} X^{-}=\sum_{r^{+}, r^{-}} \vartheta\left(\left(X^{+}\right)_{r^{+}}^{\prime,(3)}, S\left(\left(X^{-}\right)_{r^{-}}^{\prime,(3)}\right)\right) \vartheta\left(\left(X^{+}\right)_{r^{+}}^{\prime,(1)},\left(X^{-}\right)_{r^{-}}^{\prime,(1)}\right) \\
& \cdot\left(X^{-}\right)_{r^{-}}^{\prime,(2)}\left(X^{+}\right)_{r^{+}}^{\prime,(2)}
\end{aligned}
$$

for $\lambda, \mu \in \mathfrak{A}, i, j \in I$, and $X^{+}, Y^{+} \in U^{+, b}, X^{-}, Y^{-} \in U^{-, b}$, where $\left(X^{+}\right)_{k^{+}}^{(x)}$ and $\left(X^{-}\right)_{k^{-}}^{(x)}$ with $x \in J_{1,2}$ (resp. $\left(X^{+}\right)_{r^{+}}^{\prime,(y)}$ and $\left(X^{-}\right)_{r^{-}}^{\prime,(y)}$ with $\left.y \in J_{1,3}\right)$ are any elements of $U^{+, b}$ and $U^{-, b}$ respectively satisfying $\Delta\left(X^{ \pm}\right)=\sum_{k^{ \pm}}\left(X^{ \pm}\right)_{k^{ \pm}}^{(1)} \otimes\left(X^{ \pm}\right)_{k^{ \pm}}^{(2)}$, $\left(\right.$ resp. $\left.\left(\left(\operatorname{id}_{U} \otimes \Delta\right) \circ \Delta\right)\left(X^{ \pm}\right)=\sum_{r^{ \pm}}\left(X^{ \pm}\right)_{r^{ \pm}}^{\prime,(1)} \otimes\left(X^{ \pm}\right)_{r^{ \pm}}^{\prime,(2)} \otimes\left(X^{ \pm}\right)_{r^{ \pm}}^{\prime,(3)}\right)$. We have

$$
\vartheta\left(X^{+} K_{\lambda}, X^{-} L_{\mu}\right)=\chi(\lambda, \mu) \vartheta\left(X^{+}, X^{-}\right) \quad\left(\lambda, \mu \in \mathfrak{A}, X^{+} U^{+, b}, X^{-} U^{-, b}\right),
$$

and

$$
\vartheta\left(U_{\lambda}^{+}, U_{-\mu}^{-}\right)=\{0\} \quad \text { if } \lambda \neq \mu \text {. }
$$

By (2.7)-(2.8), we can easily see:

Lemma 2.1. Let $\chi: \mathfrak{A} \times \mathfrak{A} \rightarrow \mathbb{K}^{\times}$and $\pi: I \rightarrow \mathfrak{A}$ be as above. Let $\mathfrak{A}^{\prime}$ be a $\mathbb{Z}$-submodule of $\mathfrak{A}$ such that $\pi(I) \subset \mathfrak{A}^{\prime}$. Let $\mathbb{K}^{\prime}$ be a subfield of $\mathbb{K}$ such that $\chi\left(\mathfrak{A}^{\prime}, \mathfrak{A}^{\prime}\right) \subset\left(\mathbb{K}^{\prime}\right)^{\times}$. Let $\eta: \mathfrak{A}^{\prime} \rightarrow \mathfrak{A}$ be the inclusion map. Define the map $\chi^{\prime}: \mathfrak{A}^{\prime} \times \mathfrak{A}^{\prime} \rightarrow\left(\mathbb{K}^{\prime}\right)^{\times}$by $\eta \circ \chi^{\prime}=\chi \mathfrak{A}^{\prime} \times \mathfrak{A}^{\prime}$. Define the map $\pi^{\prime}: I \rightarrow \mathfrak{A}^{\prime}$ by $\eta \circ \pi^{\prime}=\pi$. Then there exists a $\mathbb{K}$-algebra monomorphism $f: U\left(\chi^{\prime}, \pi^{\prime}\right) \otimes_{\mathbb{K}^{\prime}} \mathbb{K} \rightarrow U(\chi, \pi)$ such that $f\left(K_{\lambda} L_{\mu}\right)=K_{\lambda} L_{\mu}\left(\lambda, \mu \in \mathfrak{A}^{\prime}\right)$ and $f\left(E_{i}\right)=E_{i}, f\left(F_{i}\right)=F_{i}(i \in I)$.

Define the $\mathbb{K}$-linear map $\mathfrak{S h}^{\chi, \pi}: U(\chi, \pi) \rightarrow U^{0}(\chi, \pi)$ by

$$
\mathfrak{S h}^{\chi, \pi}\left(Y K_{\lambda} L_{\mu} X\right)=\varepsilon(Y) \varepsilon(X) K_{\lambda} L_{\mu}
$$

$\left(X \in U^{+}, Y \in U^{-}, \lambda, \mu \in \mathfrak{A}\right)$.

\subsection{Kharchenko's PBW theorem}

Theorem 2.2. (Kharchenko's PBW theorem [15, Theorem 2], [16, Theorem 2.2], see also [9, Theorem 3.14] and Section 4.) Keep the notation as above. Then there exists a unique pair of $\left(R_{\chi}^{\pi,+}, \varphi_{\chi}^{\pi,+}\right)$ of a subset $R_{\chi}^{\pi,+}$ of $\mathfrak{A}_{\pi}^{+} \backslash\{0\}$ and a 
map $\varphi_{\chi}^{\pi,+}: R_{\chi}^{\pi,+} \rightarrow \mathbb{N}$ satisfying the following. Let $X:=\left\{(\alpha, t) \in R_{\chi}^{\pi,+} \times \mathbb{N} \mid t \in\right.$ $\left.J_{1, \varphi_{\chi}^{\pi,+}(\alpha)}\right\}$. Define the map $z: X \rightarrow R_{\chi}^{\pi,+}$ by $z(\alpha, t):=\alpha$. Let $Y$ be the set of maps $y: X \rightarrow \mathbb{Z}_{\geq 0}$ such that $|\{x \in X \mid y(x) \geq 1\}|<\infty$ and $(y(x))_{\chi(z(x), z(x))} ! \neq 0$ for all $x \in X$. Then

$$
\forall \lambda \in \mathfrak{A}_{\pi}^{+}, \operatorname{dim} U^{+}(\chi, \pi)_{\lambda}=\left|\left\{y \in Y \mid \sum_{x \in X} y(x) z(x)=\lambda\right\}\right| .
$$

Theorem 2.3. ([8, Proposition 1], [12, Theorem 4.9]) If $\left|R_{\chi}^{\pi,+}\right|<\infty$, then $\varphi_{\chi}^{\pi,+}\left(R_{\chi}^{\pi,+}\right)=\{1\}$.

\subsection{Skew centers}

Let $\omega: \mathfrak{A}_{\pi} \rightarrow \mathbb{K}^{\times}$be a $\mathbb{Z}$-module homomorphism.

$$
\mathfrak{Z}_{\omega}(\chi, \pi):=\left\{Z \in U(\chi, \pi)_{0} \mid \forall \lambda \in \mathfrak{A}_{\pi}, \forall X \in U(\chi, \pi)_{\lambda}, Z X=\omega(\lambda) X Z\right\} .
$$

Define the $\mathbb{Z}$-module homomorphism $\hat{\rho}^{\chi, \pi}: \mathfrak{A}_{\pi} \rightarrow \mathbb{K}^{\times}$by

$$
\hat{\rho}^{\chi, \pi}\left(\alpha_{j}\right):=\chi\left(\alpha_{j}, \alpha_{j}\right) \quad(j \in I),
$$

where $\alpha_{j}:=\pi(j)$, as above.

For each $\beta \in R_{\chi}^{\pi,+}$, let $\mathfrak{B}_{\omega}^{\chi, \pi}(\beta)$ be the $\mathbb{K}$-linear subspace of $U^{0}(\chi, \pi)$ formed by the elements

$$
\sum_{(\lambda, \mu) \in \mathfrak{A}^{2}} a_{(\lambda, \mu)} K_{\lambda} L_{\mu}
$$

with $a_{(\lambda, \mu)} \in \mathbb{K}$ satisfying the following equations $(e 1)_{\beta}-(e 4)_{\beta}$. In $(e 1)_{\beta^{-}}(e 4)_{\beta}$, let $q_{\beta}:=\chi(\beta, \beta), c_{\beta}:=\hat{o}\left(q_{\beta}\right)$ and $\omega_{\lambda, \mu ; \beta}^{\chi}:=\omega(\beta) \cdot \frac{\chi(\beta, \mu)}{\chi(\lambda, \beta)}$.

$(e 1)_{\beta}$ For $(\lambda, \mu) \in \mathfrak{A}^{2}$ and $t \in \mathbb{Z} \backslash\{0\}$, if $q_{\beta} \neq 1, c_{\beta}=0$ and $\omega_{\lambda, \mu ; \beta}^{\chi}=q_{\beta}^{t}$, then the equation $a_{(\lambda+t \beta, \mu-t \beta)}=\hat{\rho}^{\chi, \pi}(\beta)^{t} \cdot a_{(\lambda, \mu)}$ holds.

$(e 2)_{\beta}$ For $(\lambda, \mu) \in \mathfrak{A}^{2}$, if $c_{\beta}=0$ and $\omega_{\lambda, \mu ; \beta}^{\chi} \neq q_{\beta}^{t}$ for all $t \in \mathbb{Z}$, then the equation $a_{(\lambda, \mu)}=0$ holds.

$(e 3)_{\beta}$ For $(\lambda, \mu) \in \mathfrak{A}^{2}$, if $q_{\beta} \neq 1, c_{\beta} \geq 2$ and $\omega_{\lambda, \mu ; \beta}^{\chi}=q_{\beta}^{t}$ for some $t \in J_{1, c_{\beta}-1}$, the equation

$$
\begin{aligned}
& \sum_{x=-\infty}^{\infty} a_{\left(\lambda+\left(c_{\beta} x+t\right) \beta, \mu-\left(c_{\beta} x+t\right) \beta\right)} \hat{\rho}^{\chi, \pi}(\beta)^{-\left(c_{\beta} x+t\right)} \\
& =\sum_{y=-\infty}^{\infty} a_{\left(\lambda+c_{\beta} y \beta, \mu-c_{\beta} y \beta\right)} \hat{\rho}^{\chi, \pi}(\beta)^{-c_{\beta} y}
\end{aligned}
$$


holds.

$(e 4)_{\beta}$ For $(\lambda, \mu) \in \mathfrak{A}^{2}$, if $c_{\beta} \geq 2$ and $\omega_{\lambda, \mu ; \beta}^{\chi} \neq q_{\beta}^{m}$ for all $m \in \mathbb{Z}$, then the $c_{\beta}-1$ equations

$$
\begin{aligned}
& \sum_{x=-\infty}^{\infty} a_{\left(\lambda+\left(c_{\beta} x+t\right) \beta, \mu-\left(c_{\beta} x+t\right) \beta\right)} \hat{\rho}^{\chi, \pi}(\beta)^{-\left(c_{\beta} x+t\right)} \\
& \quad=\sum_{y=-\infty}^{\infty} a_{\left(\lambda+c_{\beta} y \beta, \mu-c_{\beta} y \beta\right)} \hat{\rho}^{\chi, \pi}(\beta)^{-c_{\beta} y} \\
& \left(t \in J_{1, c_{\beta}-1}\right)
\end{aligned}
$$

hold. Let

$$
\mathfrak{B}_{\omega}^{\chi, \pi}:=\bigcap_{\beta \in R_{\chi}^{\pi,+}} \mathfrak{B}_{\omega}^{\chi, \pi}(\beta) .
$$

Theorem 2.4. ([5, Theorem 10.4]) Assume $\mathfrak{A}=\mathfrak{A}_{\pi}$. Assume $\left|R_{\chi}^{\pi,+}\right|<\infty$. Assume that $\chi(\alpha, \alpha) \neq 1$ for all $\alpha \in R_{\chi}^{\pi,+}$. Then we have the $\mathbb{K}$-linear isomorphism $\mathfrak{H C}_{\omega}^{\chi, \pi}: \mathfrak{Z}_{\omega}(\chi, \pi) \rightarrow \mathfrak{B}_{\omega}^{\chi, \pi}$ defined by $\mathfrak{H}_{\omega}^{\chi,} \mathfrak{\omega}^{\chi, \pi}(X):=\mathfrak{S h}^{\chi, \pi}(X)$.

The statement of [5., Theorem 10.4] has claimed that the above theorem holds if $\mathbb{K}$ is an algebraically closed field. However, by the argument in [5], we can easily see that it really holds for any field.

\section{Elements of $\mathfrak{Z}_{\omega}(\chi, \pi)$ via finite dimensional rep- resentations}

In Subsection 3, we use argument similar to that in [19, Sections 2 and 3].

\subsection{Hopf pairing}

In Subsection 3.1, let

$$
\mathrm{A}=\left(\mathrm{A}, \Delta_{\mathrm{A}}, S_{\mathrm{A}}, \varepsilon_{\mathrm{A}}\right) \text { and } \mathrm{B}=\left(\mathrm{B}, \Delta_{\mathrm{B}}, S_{\mathrm{B}}, \varepsilon_{\mathrm{B}}\right)
$$

be Hopf algebras over $\mathbb{K}$ with $S_{\mathrm{A}}$ and $S_{\mathrm{B}}$ being bijective, and assume that there exists a Hopf pairing $\mathrm{p}: \mathrm{A} \times \mathrm{B} \rightarrow \mathbb{K}$, that is, $\mathrm{p}\left(a_{1} a_{2}, b\right)=\mathrm{p}\left(a_{1} \otimes a_{2}, \Delta_{\mathrm{B}}(b)\right)$, $\mathrm{p}\left(a, b_{1} b_{2}\right)=\mathrm{p}\left(\Delta_{\mathrm{A}}(a), b_{1} \otimes b_{2}\right), \mathrm{p}\left(S_{\mathrm{A}}(a), b\right)=\mathrm{p}\left(a, S_{\mathrm{B}}(b)\right), \mathrm{p}\left(a, 1_{\mathrm{B}}\right)=\varepsilon_{\mathrm{A}}(a)$, and $\mathrm{p}\left(1_{\mathrm{A}}, b\right)=\varepsilon_{\mathrm{B}}(b)$ hold for all $a, a_{1}, a_{2} \in \mathrm{A}$ and all $b, b_{1}, b_{2} \in \mathrm{B}$, where $\mathrm{p}\left(a_{1} \otimes\right.$ $\left.a_{2}, b_{1} \otimes b_{2}\right):=p\left(a_{1}, b_{1}\right) p\left(a_{2}, b_{2}\right)$. Note that from $\mathrm{B}$, we obtain the Hopf algebra $\mathrm{B}^{\mathrm{op}}=\left(\mathrm{B}, y \circ \Delta_{\mathrm{B}}, S_{\mathrm{B}}^{-1}, \varepsilon_{\mathrm{B}}\right)$, where we define the $\mathbb{K}$-linear isomorphism $y: \mathrm{B} \otimes \mathrm{B} \rightarrow$ $\mathrm{B} \otimes \mathrm{B}$ by $y\left(b_{1} \otimes b_{2}\right):=b_{2} \otimes b_{1}$, i.e., $\mathrm{B}^{\text {op }}$ is the opposite Hopf algebra of $\mathrm{B}$.

It is well-known that we have the Hopf algebra $\mathrm{D}=\mathrm{D}(\mathrm{p}, \mathrm{A}, \mathrm{B})=\left(\mathrm{D}, \Delta_{\mathrm{D}}, S_{\mathrm{D}}, \varepsilon_{\mathrm{D}}\right)$ satisfying the conditions below. 
(D1) D include $A$ and $B^{o p}$ as Hopf subalgebras. The $\mathbb{K}$-linear homomorphism $\mathrm{A} \otimes \mathrm{B} \rightarrow \mathrm{D}, a \otimes b \mapsto a b$, is bijective.

(D2) As a $\mathbb{K}$-algebra, D has the multiplication such that for $a \in \mathrm{A}$ and $b \in \mathrm{B}, b a=\sum_{i, j} \mathrm{p}\left(S_{\mathrm{A}}^{-1}\left(a_{i}^{(1)}\right), b_{j}^{(3)}\right) \mathrm{p}\left(a_{i}^{(3)}, b_{j}^{(1)}\right) a_{i}^{(2)} b_{j}^{(2)}$, where $\left(\left(\Delta_{\mathrm{A}} \otimes \mathrm{id}\right) \circ \Delta_{\mathrm{A}}\right)(a)=$ $\sum_{i} a_{i}^{(1)} \otimes a_{i}^{(2)} \otimes a_{i}^{(3)}$ and $\left(\left(\Delta_{\mathrm{B}} \otimes \mathrm{id}\right) \circ \Delta_{\mathrm{B}}\right)(b)=\sum_{j} b_{j}^{(1)} \otimes b_{j}^{(2)} \otimes b_{j}^{(3)}$; we also have $a b=\sum_{i, j} \mathrm{p}\left(S_{\mathrm{A}}^{-1}\left(a_{i}^{(3)}\right), b_{j}^{(1)}\right) \mathrm{p}\left(a_{i}^{(1)}, b_{j}^{(3)}\right) b_{j}^{(2)} a_{i}^{(2)}$.

We can easily see that the $\mathbb{K}$-linear homomorphism $\mathrm{B} \otimes \mathrm{A} \rightarrow \mathrm{D}, b \otimes a \rightarrow b a$, is bijective. Define the $\mathbb{K}$-bilinear map $\mathrm{P}: \mathrm{D} \times \mathrm{D} \rightarrow \mathbb{K}$ by

$$
\mathrm{P}\left(a_{1} b_{1}, b_{2} a_{2}\right):=\mathrm{p}\left(a_{1}, b_{2}\right) \cdot \mathrm{p}\left(a_{2}, b_{1}\right) \quad\left(a_{1}, a_{2} \in \mathrm{A}, b_{1}, b_{2} \in \mathrm{B}\right) .
$$

Define the left (resp. right) $\mathbb{K}$-algebra action $\bullet_{1}\left(\right.$ resp. $\left.\bullet_{r}\right)$ of $D$ on $D$ by

$$
x \bullet_{1} y:=\sum_{i} x_{i}^{(1)} y S_{\mathrm{D}}\left(x_{i}^{(2)}\right) \quad\left(\text { resp. } y \bullet_{\mathrm{r}} x:=\sum_{i} S_{\mathrm{D}}\left(x_{i}^{(1)}\right) y x_{i}^{(2)}\right),
$$

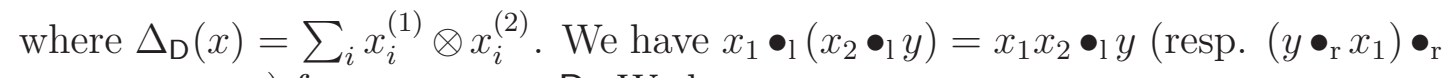
$\left.x_{2}=y \bullet_{\mathrm{r}} x_{1} x_{2}\right)$ for $x_{1}, x_{2}, y \in \mathrm{D}$. We have

Lemma 3.1. It follows that

$$
\mathrm{P}\left(x \bullet_{1} y_{1}, y_{2}\right)=\mathrm{P}\left(y_{1}, y_{2} \bullet_{\mathrm{r}} x\right) \quad\left(x, y_{1}, y_{2} \in \mathrm{D}\right) .
$$

Proof. (cf. [19, Proposition 2.2.1]) Letting $\mathbf{X}$ be $\mathrm{A}$ or $\mathrm{B}$, for $c \in \mathbf{X}$ and $r \in \mathbb{N}$ with $r \geq 2$, let

$$
\begin{aligned}
& \sum_{i} c_{i}^{(1)} \otimes \cdots \otimes c_{i}^{(r-1)} \otimes c_{i}^{(r)} \\
& \quad:=\left((\Delta_{\mathbf{X}} \otimes \underbrace{\mathrm{id} \mathrm{d}_{\mathbf{X}} \otimes \cdots \otimes \mathrm{id}_{\mathbf{X}}}_{r-2}) \circ \cdots \circ\left(\Delta_{\mathbf{X}} \otimes \mathrm{id}_{\mathbf{X}}\right) \circ \Delta_{\mathbf{X}}\right)(c) .
\end{aligned}
$$

(Note that $\Delta_{\mathrm{D}}(a)=\sum_{i} a_{i}^{(1)} \otimes a_{i}^{(2)}(a \in \mathrm{A})$, and $\left.\Delta_{\mathrm{D}}(b)=\sum_{j} b_{i}^{(2)} \otimes b_{j}^{(1)}(b \in \mathrm{B}).\right)$

Let $a, \dot{a}, \grave{a} \in \mathrm{A}$ and $b, \grave{b}, \grave{b} \in \mathrm{B}$. We have

$$
\begin{aligned}
& a \bullet \mathfrak{a} \dot{b}=\sum_{i, j} \mathrm{p}\left(a_{i}^{(4)}, \hat{b}_{j}^{(3)}\right) \mathrm{p}\left(S_{\mathrm{A}}\left(a_{i}^{(2)}\right), \dot{b}_{j}^{(1)}\right) a_{i}^{(1)} \dot{a} S_{\mathrm{A}}\left(a_{i}^{(3)}\right) \dot{b}_{j}^{(2)}, \\
& \grave{b} \grave{a} \bullet \bullet_{\mathrm{r}} a=\sum_{i, j} \mathrm{p}\left(a_{i}^{(1)}, \grave{b}_{j}^{(1)}\right) \mathrm{p}\left(S_{\mathrm{A}}\left(a_{i}^{(3)}\right), \grave{b}_{j}^{(3)}\right) \grave{b}_{j}^{(2)} S_{\mathrm{A}}\left(a_{i}^{(2)}\right) \grave{a} a_{i}^{(4)} \text {, }
\end{aligned}
$$

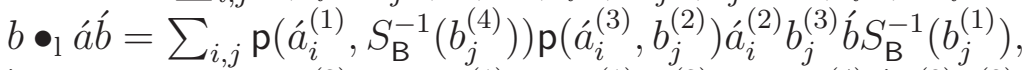

$$
\begin{aligned}
& \grave{b} \grave{a} \bullet_{\mathrm{r}} b=\sum_{i, j} \mathrm{p}\left(\grave{a}_{i}^{(3)}, S_{\mathrm{B}}^{-1}\left(b_{j}^{(1)}\right)\right) \mathrm{p}\left(\grave{a}_{i}^{(1)}, b_{j}^{(3)}\right) S_{\mathrm{B}}^{-1}\left(b_{j}^{(4)}\right) \grave{b} b_{j}^{(2)} \grave{a}_{i}^{(2)} \text {. }
\end{aligned}
$$

Hence we have

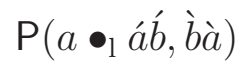

$$
\begin{aligned}
& =\sum_{i, j} \mathrm{p}\left(a_{i}^{(4)}, \dot{b}_{j}^{(3)}\right) \mathrm{p}\left(S_{\mathrm{A}}\left(a_{i}^{(3)}\right), \dot{b}_{j}^{(1)}\right) \mathrm{p}\left(a_{i}^{(1)} \dot{a} S_{\mathrm{A}}\left(a_{i}^{(3)}\right), \grave{b}\right) \mathrm{p}\left(\grave{a}, \dot{b}_{j}^{(2)}\right) \\
& =\sum_{i} \mathrm{p}\left(S_{\mathrm{A}}\left(a_{i}^{(3)}\right) \grave{a} a_{i}^{(4)}, \hat{b}\right) \mathrm{p}\left(a_{i}^{(1)} \dot{a} S_{\mathrm{A}}\left(a_{i}^{(3)}\right), \grave{b}\right) \\
& =\sum_{i, j} \mathrm{p}\left(S_{\mathrm{A}}\left(a_{i}^{(3)}\right) \grave{a} a_{i}^{(4)}, \hat{b}\right) \mathrm{p}\left(a_{i}^{(1)}, \grave{b}_{j}^{(1)}\right) \mathrm{p}\left(S_{\mathrm{A}}\left(a_{i}^{(3)}\right), \grave{b}_{j}^{(3)}\right) \mathrm{p}\left(\grave{a}, \grave{b}_{j}^{(2)}\right) \\
& =\mathrm{P}\left(\dot{a} \hat{b}, \grave{b} \grave{a} \bullet_{\mathrm{r}} a\right) \text {, }
\end{aligned}
$$


and

$$
\begin{aligned}
\mathrm{P} & \left(b \bullet_{1} \dot{a} \dot{b}, \grave{b} \grave{a}\right) \\
& =\sum_{i, j} \mathrm{p}\left(\dot{a}_{i}^{(1)}, S_{\mathrm{B}}^{-1}\left(b_{j}^{(4)}\right)\right) \mathrm{p}\left(\dot{a}_{i}^{(3)}, b_{j}^{(2)}\right) \mathrm{p}\left(\dot{a}_{i}^{(2)}, \grave{b}\right) \mathrm{p}\left(\grave{a}, b_{j}^{(3)} \dot{b} S_{\mathrm{B}}^{-1}\left(b_{j}^{(1)}\right)\right) \\
& =\sum_{j} \mathrm{p}\left(\grave{a}, S_{\mathrm{B}}^{-1}\left(b_{j}^{(4)}\right) \grave{b} b_{j}^{(2)}\right) \mathrm{p}\left(\grave{a}, b_{j}^{(3)} \grave{b} S_{\mathrm{B}}^{-1}\left(b_{j}^{(1)}\right)\right) \\
& =\sum_{i, j} \mathrm{p}\left(\grave{a}, S_{\mathrm{B}}^{-1}\left(b_{j}^{(4)}\right) \grave{b} b_{j}^{(2)}\right) \mathrm{p}\left(\grave{a}_{i}^{(1)}, b_{j}^{(3)}\right) \mathrm{p}\left(\grave{a}_{i}^{(2)}, \grave{b}\right) \mathrm{p}\left(\grave{a}_{i}^{(3)}, S_{\mathrm{B}}^{-1}\left(b_{j}^{(1)}\right)\right) \\
& =\mathrm{P}\left(\dot{a} \grave{b}, \grave{b} \grave{a} \bullet_{\mathrm{r}} b\right) .
\end{aligned}
$$

This completes the proof.

Let $\mathrm{D}^{*}$ be the $\mathbb{K}$-linear space formed by all $\mathbb{K}$-linear homomorphisms from $\mathrm{D}$ to $\mathbb{K}$. Define the right $\mathbb{K}$-algebra action · of $\mathrm{D}$ on $\mathrm{D}^{*}$ by

$$
(f \cdot x)(y):=f(x \bullet y) \quad\left(f \in \mathrm{D}^{*}, x, y \in \mathrm{D}\right) .
$$

Define the $\mathbb{K}$-linear homomorphism $\Omega_{\mathrm{D}}: \mathrm{D} \rightarrow \mathrm{D}^{*}$ by $\left(\Omega_{\mathrm{D}}(x)\right)(y):=\mathrm{P}(y, x)$ $(x, y \in \mathrm{D})$. By (3.1), we have

$$
\Omega_{\mathrm{D}}(y) \cdot x=\Omega_{\mathrm{D}}\left(y \bullet \bullet_{\mathrm{r}} x\right) \quad(x, y \in \mathrm{D}) .
$$

Lemma 3.2. Assume that $\mathrm{p}$ is non-degenerate. Let $x, y \in \mathrm{D}$. Then

$$
\varepsilon_{\mathrm{D}}(y) x=x \bullet_{\mathrm{r}} y \quad \Longleftrightarrow \quad \Omega_{\mathrm{D}}(x) \cdot y=\varepsilon_{\mathrm{D}}(y) \Omega_{\mathrm{D}}(x) .
$$

Proof. Since $\mathrm{p}$ is non-degenerate, $\mathrm{P}$ is so. Hence $\Omega_{\mathrm{D}}$ is injective. Then (3.3) follows from (3.2).

\subsection{Extension of $U=U(\chi, \pi)$}

Keep the notation in Subsection 2.2, Recall $I=J_{1, l}$ and $\alpha_{i}=\pi(i)(i \in I)$. Let $\breve{I}:=J_{1,2 l+2}$, i.e., $\breve{I}=I \cup J_{l+1,2 l+2}$. Let $\alpha_{j} \in \mathfrak{A}\left(j \in J_{l+1,2 l+2}\right)$. Let $\omega: \mathfrak{A}_{\pi} \rightarrow \mathbb{K}^{\times}$ be a $\mathbb{Z}$-module homomorphism.

In Subsections 3.2 and 3.3, we assume the following (ass1)-(ass4).

(ass1) There exists $\xi \in \mathbb{K}^{\times} \backslash\{1\}$ with $\hat{o}(\xi)=0$.

(ass2) $\mathfrak{A}$ is a $\mathbb{Z}$-module with $\operatorname{rank}_{\mathbb{Z}} \mathfrak{A}=2 l+2$ and $\mathfrak{A}=\bigoplus_{i \in \breve{I}} \mathbb{Z} \alpha_{i}$.

(ass3) One has $\chi\left(\alpha_{i}, \alpha_{j+l+1}\right)=\chi\left(\alpha_{j+l+1}, \alpha_{i}\right)=\xi^{\delta_{i j}}$ and $\chi\left(\alpha_{i+l+1}, \alpha_{j+l+1}\right)=1$ for $i, j \in J_{1, l+1}$.

(ass4) One has $\omega\left(\alpha_{i}\right)=\chi\left(\alpha_{i}, \alpha_{l+1}\right)$ and $\chi\left(\alpha_{l+1}, \alpha_{i}\right)=1$ for $i \in I$.

Let $X$ and $Y$ be a $\mathbb{K}$-linear space, and let $f: X \times Y \rightarrow \mathbb{K}$ be a a $\mathbb{K}$-bilinear map. We call $\mathbb{K}$-linear space $\{x \in X \mid \forall y \in Y, f(x, y)=0\}$ (resp. $\{y \in Y \mid \forall x \in$ $X, f(x, y)=0\}$ ) of $X$ (resp. $Y$ ) the left kernel (resp. the right kernel). We call $f$ non-degenerate if the right and left kernels are zero-dimensional. 
Lemma 3.3. Let $U^{+, b, 0}:=\oplus_{\lambda \in \mathfrak{A}} \mathbb{K} K_{\lambda}\left(=U^{0} \cap U^{+, b}\right)$ and $U^{-, b, 0}:=\oplus_{\lambda \in \mathfrak{A} A} \mathbb{K} L_{\lambda}(=$ $\left.U^{0} \cap U^{-, b}\right)$. Then $\vartheta_{\mid U^{+, b, 0} \times U^{-, b, 0}}$ is non-degenerate. In particular, $\vartheta$ is nondegenerate.

Proof. Let $\vartheta^{\prime}:=\vartheta_{\mid U^{+, b, 0} \times U^{-, b, 0}}$. Let $\mathfrak{A}^{\prime}:=\oplus_{i=1}^{l} \mathbb{Z} \alpha_{i}$ and $\mathfrak{A}^{\prime \prime}:=\oplus_{j=1}^{l} \mathbb{Z} \alpha_{j+l}$. So $\mathfrak{A}=\mathfrak{A}^{\prime} \oplus \mathfrak{A}^{\prime \prime}$. Let $\left(U^{-, b, 0}\right)^{\prime}:=\oplus_{\lambda \in \mathfrak{A}^{\prime}} \mathbb{K} L_{\lambda}$ and $\left(U^{+, b, 0}\right)^{\prime \prime}:=\oplus_{\mu \in \mathfrak{Q}^{\prime \prime}} \mathbb{K} K_{\mu}$. Let $R$ (resp. $R^{\prime}$ ) be the left (resp. right) kernel of $\vartheta^{\prime}$. Let $r \in R$. Write $r=\sum_{t=1}^{z} r_{t}$ for some $z \in \mathbb{N}$ and $\lambda_{t} \in \mathfrak{A}^{\prime}\left(t \in J_{1, z}\right)$ with $r_{t} \in K_{\lambda_{t}}\left(U^{+, b, 0}\right)^{\prime \prime}\left(t \in J_{1, z}\right)$. For $t \in J_{1, z}$, write $\lambda_{t}=\sum_{i=1}^{l} x_{i}^{(t)} \alpha_{i}$ with $x_{i}^{(t)} \in \mathbb{Z}$. Since $\vartheta^{\prime}\left(r, h L_{\alpha_{l+1}}^{y_{1}} \cdots L_{\alpha_{2 l}}^{y_{l}}\right)=0$ and $\vartheta^{\prime}\left(r_{t}, h L_{\alpha_{l+1}}^{y_{1}} \cdots L_{\alpha_{2 l}}^{y_{l}}\right)=\xi^{x_{1}^{(t)} y_{1}+\cdots+x_{l}^{(t)} y_{l}} \vartheta^{\prime}\left(r_{t}, h\right)$ for all $h \in\left(U^{-, b, 0}\right)^{\prime}$ and all $y_{i} \in \mathbb{Z}$ $\left(i \in J_{1, l}\right)$, we have $r_{t} \in R$ for all $t \in J_{1, z}$. Since $\vartheta^{\prime}\left(r_{t},\left(U^{-, b, 0}\right)^{\prime}\right)=\{0\}$, we have $r_{t}=0$. Hence $r=0$. Hence $R=\{0\}$. Similarly we have $R^{\prime}=\{0\}$.

We have the Hopf algebra isomorphism $\mathrm{D}\left(\vartheta, U^{+},\left(U^{-}\right)^{\text {op }}\right) \rightarrow U, X \otimes Y \rightarrow X Y$.

From now on until the end of Section 3, we identify $U$ with $\mathrm{D}$, let the actions $\bullet_{\mathrm{r}}, \bullet_{1}, \cdot$ of $U$ be the ones defined for $U$ by identifying $\vartheta$ with $\mathrm{p}$.

\subsection{Central elements from finite dimensional modules un- der the assumption (3.4)}

Keep the notation as in Subsection 3.2. We have assumed (3.4). From now on until the end of this subsection

$$
\text { fix } \lambda, \mu \in \mathfrak{A} \text {, }
$$

and fix a non-zero $U$-module $\breve{V}$ such that there exists $\breve{v} \in \breve{V}$ with

$$
K_{\lambda^{\prime}} L_{\mu^{\prime}} \breve{v}:=\chi\left(\lambda, \mu^{\prime}\right) \chi\left(\lambda^{\prime}, \mu\right) \breve{v}\left(\lambda^{\prime}, \mu^{\prime} \in \mathfrak{A}\right) \text { and } F_{i} \breve{v}:=0(i \in I) .
$$

Define the $\mathbb{K}$-algebra homomorphism $\breve{a}: U \rightarrow \operatorname{End}_{\mathbb{K}}(\breve{V})$ by $\breve{a}(X)(h):=X h$ $(X \in U, h \in \breve{V})$, i.e., $(\breve{a}, \breve{V})$ is the $\mathbb{K}$-algebra representation of $U$ associated with $\breve{V}$. By Lemma 3.3, we have $\breve{V}=\oplus_{\nu \in \mathfrak{A}_{\pi}^{+}} U_{-\nu}^{-} \breve{v}$, as a $\mathbb{K}$-linear space, and

$$
U_{-\nu}^{-} \breve{v}=\left\{v \in \breve{V} \mid K_{\lambda^{\prime}} L_{\mu^{\prime}} v=\chi\left(\lambda+\nu, \mu^{\prime}\right) \chi\left(\lambda^{\prime}, \mu-\nu\right) v\left(\lambda^{\prime}, \mu^{\prime} \in \mathfrak{A}\right)\right\} .
$$

For $\nu \in \mathfrak{A}_{\pi}^{+}$, let $\breve{m}_{\nu}:=\operatorname{dim} U_{-\nu}^{-} \breve{v}$.

$$
\text { Assume } \operatorname{dim}_{\mathbb{K}} \breve{V}<\infty \text {. }
$$

Then $\operatorname{dim}_{\mathbb{K}} \breve{V}=\sum_{\nu \in \mathfrak{A}_{\pi}^{+}} \breve{m}_{\nu}$. For $k \in \operatorname{End}_{\mathbb{K}}(\breve{V})$, let $\operatorname{Tr}_{\breve{V}}(k) \in \mathbb{K}$ denote the trace of $k$. Define $\breve{\rho} \in \operatorname{End}_{\mathbb{K}}(\breve{V})$ by $\breve{\rho}(Y \breve{v}):=\hat{\rho}^{\chi, \pi}(\nu) Y \breve{v}$ for $\nu \in \mathfrak{A}_{\pi}^{+}$and $Y \in U_{-\nu}^{-}$. Since $S^{2}(X)=\hat{\rho}^{\chi, \pi}\left(-\lambda^{\prime}\right) X\left(\lambda^{\prime} \in \mathfrak{A}, X \in U_{\lambda^{\prime}}\right)$, we have

$$
S^{2}\left(Y_{1}\right) \breve{\rho}\left(Y_{2} \breve{v}\right)=\breve{\rho}\left(Y_{1} Y_{2} \breve{v}\right) \quad\left(Y_{1}, Y_{2} \in U\right) .
$$

Define $\breve{f} \in U^{*}$ by $\breve{f}(X):=\operatorname{Tr}_{\breve{V}}(\breve{a}(X) \circ \breve{\rho})(X \in U)$. 
Lemma 3.4. We have

$$
\breve{f} \cdot X=\varepsilon(X) \breve{f} \quad(X \in U) .
$$

Proof. Let $X, Y \in U$. Write $\Delta(X)=\sum_{r} X_{r}^{(1)} \otimes X_{r}^{(2)}$. Then

$$
\begin{aligned}
(\breve{f} \cdot X)(Y) & =\breve{f}\left(X \bullet_{1} Y\right) \\
& =\sum_{r} \breve{f}\left(X_{r}^{(1)} Y S\left(X_{r}^{(2)}\right)\right) \\
& =\sum_{r} \operatorname{Tr}_{\breve{V}}\left(\breve{a}\left(X_{r}^{(1)} Y S\left(X_{r}^{(2)}\right)\right) \circ \breve{\rho}\right) \\
& =\sum_{r} \operatorname{Tr}_{\breve{V}}\left(\breve{a}\left(Y S\left(X_{r}^{(2)}\right)\right) \circ \breve{\rho} \circ \breve{a}\left(X_{r}^{(1)}\right)\right) \\
& =\sum_{r} \operatorname{Tr}_{\breve{V}}\left(\breve{a}\left(Y S\left(X_{r}^{(2)}\right)\right) \circ \breve{a}\left(S^{2}\left(X_{r}^{(1)}\right)\right) \circ \breve{\rho}\right) \\
& =\sum_{r} \operatorname{Tr}_{\breve{V}}\left(\breve{a}\left(Y S\left(X_{r}^{(2)}\right) S^{2}\left(X_{r}^{(1)}\right)\right) \circ \breve{\rho}\right) \\
& =\sum_{r} \operatorname{Tr}_{\breve{V}}\left(\breve{a}\left(Y S\left(S\left(X_{r}^{(1)}\right) X_{r}^{(2)}\right)\right) \circ \breve{\rho}\right) \\
& =\varepsilon(X) \operatorname{Tr}_{\breve{V}}(\breve{a}(Y) \circ \breve{\rho}) \\
& =\varepsilon(X) \breve{f}(Y) .
\end{aligned}
$$

This completes the proof.

Define the $\mathbb{Z}$-module homomorphism $\breve{\omega}_{0}: \mathfrak{A}_{\pi} \rightarrow \mathbb{K}^{\times}$by $\breve{\omega}_{0}\left(\mathfrak{A}_{\pi}\right):=\{1\}$. Then we can easily see the equations below.

$$
\begin{aligned}
\mathfrak{Z}_{\breve{\omega}_{0}}(\chi, \pi) & =\{X \in U \mid X Y=Y X(Y \in U)\} \\
& =\left\{X \in U \mid \varepsilon(Y) X=X \bullet_{\mathrm{r}} Y(Y \in U)\right\} .
\end{aligned}
$$

Proposition 3.5. We have

$$
\breve{f} \in \Omega_{U}\left(\mathfrak{Z}_{\breve{\omega}_{0}}(\chi, \pi)\right)
$$

and

$$
\mathfrak{H} \mathfrak{C}_{\breve{\omega}_{0}}^{\chi, \pi}\left(\Omega_{U}^{-1}(\breve{f})\right)=\sum_{\nu \in \mathfrak{A}_{\pi}^{+}} \hat{\rho}^{\chi, \pi}(\nu) \breve{m}_{\nu} K_{\lambda+\nu} L_{\mu-\nu}
$$

Proof. For $\nu \in \mathfrak{A}_{\pi}^{+}$, let $m_{\nu}:=\operatorname{dim} U_{\nu}^{+}$, let $\left\{X_{\nu, x} \mid x \in J_{1, m_{\nu}}\right\}$ and $\left\{Y_{-\nu, y} \mid y \in\right.$ $\left.J_{1, m_{\nu}}\right\}$ be $\mathbb{K}$-base of $U_{\nu}^{+}$and $U_{-\nu}^{-}$respectively such that $\breve{\vartheta}\left(X_{\nu, x}, Y_{-\lambda^{\prime}, y}\right)=\delta_{\nu, \lambda^{\prime}} \delta_{x, y}$ $\left(\lambda^{\prime} \in \mathfrak{A}_{\pi}^{+}\right)$, and let $\breve{Y}_{-\nu, z} \in U_{-\nu}^{-}\left(z \in J_{1, \breve{m}_{\nu}}\right)$ be such that $\left\{\breve{Y}_{-\nu, z} \breve{v} \mid z \in J_{1, \breve{m}_{\nu}}\right\}$

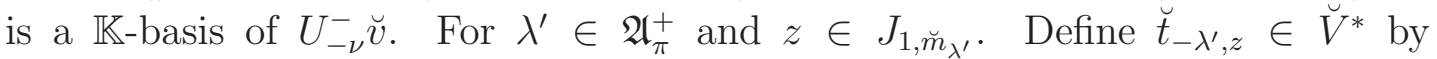
$\breve{t}_{-\lambda^{\prime}, z}\left(\breve{Y}_{-\nu, y} \breve{v}\right)=\delta_{\lambda^{\prime}, \nu} \delta_{z, y}$. 
For $\lambda^{\prime}, \mu^{\prime} \in \mathfrak{A}_{\pi}^{+}, x \in J_{1, m_{\lambda^{\prime}}}, y \in J_{1, m_{\mu^{\prime}}}$ and $\lambda^{\prime \prime}, \mu^{\prime \prime} \in \mathfrak{A}$, we have

$$
\begin{aligned}
& \breve{f}\left(X_{\lambda^{\prime}, x} K_{\lambda^{\prime \prime}} Y_{-\mu^{\prime}, y} L_{\mu^{\prime \prime}}\right) \\
& =\sum_{\nu \in \mathfrak{A}_{\pi}^{+}} \sum_{z=1}^{m_{\nu}} \breve{t}_{-\nu, z}\left(X_{\lambda^{\prime}, x} K_{\lambda^{\prime \prime}} Y_{-\mu^{\prime}, y} L_{\mu^{\prime \prime}} \breve{\rho}\left(\breve{Y}_{-\nu, z} \breve{v}\right)\right) \\
& =\sum_{\nu \in \mathfrak{A}_{\pi}^{+}} \sum_{z=1}^{m_{\nu}} \hat{\rho}^{\chi, \pi}(\nu) \breve{t}_{-\nu, z}\left(X_{\lambda^{\prime}, x} K_{\lambda^{\prime \prime}} Y_{-\mu^{\prime}, y} L_{\mu^{\prime \prime}} \breve{Y}_{-\nu, z} \breve{v}\right) \\
& =\sum_{\nu \in \mathfrak{A}_{\pi}^{+}} \sum_{z=1}^{m_{\nu}} \hat{\rho}^{\chi, \pi}(\nu) \chi\left(\lambda^{\prime \prime},-\mu^{\prime}-\nu+\mu\right) \chi\left(\nu+\lambda, \mu^{\prime \prime}\right) \\
& \cdot \breve{t}_{-\nu, z}\left(X_{\lambda^{\prime}, x} Y_{-\mu^{\prime}, y} \breve{Y}_{-\nu, z} \breve{v}\right) \\
& =\delta_{\lambda^{\prime}, \mu^{\prime}} \sum_{\nu \in \mathfrak{A}_{\pi}^{+}} \sum_{z=1}^{m_{\nu}} \hat{\rho}^{\chi, \pi}(\nu) \chi\left(\lambda^{\prime \prime},-\lambda^{\prime}-\nu+\mu\right) \chi\left(\nu+\lambda, \mu^{\prime \prime}\right) \\
& \cdot \breve{t}_{-\nu, z}\left(X_{\lambda^{\prime}, x} Y_{-\lambda^{\prime}, y} \breve{Y}_{-\nu, z} \breve{v}\right) \\
& =\delta_{\lambda^{\prime}, \mu^{\prime}} \sum_{\nu \in \mathfrak{A}_{\pi}^{+}} \sum_{z=1}^{m_{\nu}} \hat{\rho}^{\chi, \pi}(\nu) \breve{t}_{-\nu, z}\left(X_{\lambda^{\prime}, x} Y_{-\lambda^{\prime}, y} \breve{Y}_{-\nu, z} \breve{v}\right) \\
& \cdot \Omega_{U}\left(Y_{-\lambda^{\prime}, x} L_{-\lambda^{\prime}-\nu+\mu} X_{\mu^{\prime}, y} K_{\nu+\lambda}\right)\left(X_{\lambda^{\prime}, x} K_{\lambda^{\prime \prime}} Y_{-\mu^{\prime}, y} L_{\mu^{\prime \prime}}\right) \\
& =\sum_{\nu \in \mathfrak{A}_{\pi}^{+}} \sum_{z=1}^{m_{\nu}} \hat{\rho}^{\chi, \pi}(\nu) \breve{t}_{-\nu, z}\left(X_{\lambda^{\prime}, x} Y_{-\lambda^{\prime}, y} \breve{Y}_{-\nu, z} \breve{v}\right) \\
& \cdot \Omega_{U}\left(Y_{-\lambda^{\prime}, x} L_{-\lambda^{\prime}-\nu+\mu} X_{\lambda^{\prime}, y} K_{\nu+\lambda}\right)\left(X_{\lambda^{\prime}, x} K_{\lambda^{\prime \prime}} Y_{-\mu^{\prime}, y} L_{\mu^{\prime \prime}}\right) \\
& =\sum_{\nu, \nu^{\prime} \in \mathfrak{A}_{\pi}^{+}} \sum_{x^{\prime}, y^{\prime}=1}^{m_{\prime^{\prime}}} \sum_{z=1}^{m_{\nu}} \hat{\rho}^{\chi, \pi}(\nu) \breve{t}_{-\nu, z}\left(X_{\nu^{\prime}, x} Y_{-\nu^{\prime}, y} \breve{Y}_{-\nu, z} \breve{v}\right) \\
& \cdot \Omega_{U}\left(Y_{-\nu^{\prime}, x^{\prime}} L_{-\nu^{\prime}-\nu+\mu} X_{\nu^{\prime}, y^{\prime}} K_{\nu+\lambda}\right)\left(X_{\lambda^{\prime}, x} K_{\lambda^{\prime \prime}} Y_{-\mu^{\prime}, y} L_{\mu^{\prime \prime}}\right) \\
& =\Omega_{U}\left(\sum_{\nu, \nu^{\prime} \in \mathfrak{A}_{\pi}^{+}} \sum_{x^{\prime}, y^{\prime}=1}^{m_{\nu^{\prime}}} \sum_{z=1}^{m_{\nu}} \hat{\rho}^{\chi, \pi}(\nu) \breve{t}_{-\nu, z}\left(X_{\nu^{\prime}, x} Y_{-\nu^{\prime}, y} \breve{Y}_{-\nu, z} \breve{v}\right)\right. \\
& \left.\cdot Y_{-\nu^{\prime}, x^{\prime}} L_{-\nu^{\prime}-\nu+\mu} X_{\nu^{\prime}, y^{\prime}} K_{\nu+\lambda}\right)\left(X_{\lambda^{\prime}, x} K_{\lambda^{\prime \prime}} Y_{-\mu^{\prime}, y} L_{\mu^{\prime \prime}}\right) \text {. }
\end{aligned}
$$

Hence $\breve{f} \in \operatorname{Im} \Omega_{U}$ and

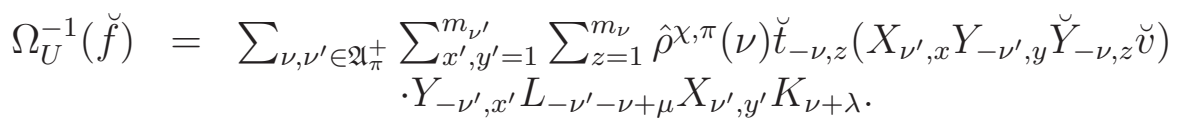

By (3.3), (3.5) and (3.6), we have $\Omega_{U}^{-1}(\breve{f}) \in \mathfrak{Z}_{\breve{\omega}_{0}}(\chi, \pi)$, which implies (3.7). By (3.10), we have (3.8). This completes the proof.

Let $U_{\pi}^{0}=U_{\pi}^{0}(\chi, \pi):=\oplus_{\nu, \nu^{\prime} \in \mathfrak{A}_{\pi}} \mathbb{K} K_{\nu} L_{\nu^{\prime}}$ and $U_{\pi}=U_{\pi}(\chi, \pi):=\operatorname{Span}_{\mathbb{K}}\left(U^{-} U_{\pi}^{0} U^{+}\right)$. Then $U_{\pi}$ (resp. $U_{\pi}^{0}$ ) is a $\mathbb{K}$-subalgebra of $U$ (resp. $\left.U^{0}\right)$.

Lemma 3.6. Assume $\lambda, \mu-\alpha_{l+1} \in \mathfrak{A}_{\pi}$. Let $Z:=\Omega_{U}^{-1}(\breve{f}) L_{-\alpha_{l+1}}$. Then $Z \in$ $U_{\pi} \cap \mathfrak{Z}_{\omega}(\chi, \pi)$.

Proof. This easily follows from (3.10).

\subsection{Conjecture on a basis of $\mathfrak{Z}_{\omega}(\chi, \pi)$}

In this subsection, assume $\mathfrak{A}=\mathfrak{A}_{\pi}$. Then $U=U_{\pi}(\chi, \pi)$. Let $\omega: \mathfrak{A} \rightarrow \mathbb{K}^{\times}$be a $\mathbb{Z}$-module homomorphism. For a $\mathbb{K}$-algebra homomorphism $\Lambda: U^{0} \rightarrow \mathbb{K}$, there exists a simple $U$-module $\mathcal{L}(\Lambda)$ such that there exists $v_{\Lambda} \in \mathcal{L}(\Lambda) \backslash\{0\}$ such that $\mathcal{L}(\Lambda)=\oplus_{\nu \in \mathfrak{A}_{\pi}^{+}} U_{-\nu}^{-} v_{\Lambda}, Z v_{\Lambda}=\Lambda(Z) v_{\Lambda}$ and $E_{i} v_{\Lambda}=0(i \in I)$.

For $\lambda, \mu \in \mathfrak{A}$, define the $\mathbb{K}$-algebra homomorphism $\Lambda_{\lambda, \mu ; \omega}^{\chi}: U^{0} \rightarrow \mathbb{K}$ by

$$
\Lambda_{\lambda, \mu ; \omega}^{\chi}\left(K_{\lambda^{\prime}} L_{\mu^{\prime}}\right):=\chi\left(\lambda, \mu^{\prime}\right) \chi\left(\lambda^{\prime}, \mu\right) \omega\left(\lambda^{\prime}\right) \quad\left(\lambda^{\prime}, \mu^{\prime} \in \mathfrak{A}\right) .
$$


Let

$$
\operatorname{Fin}_{\omega}^{\chi}:=\left\{(\lambda, \mu) \in \mathfrak{A} \times \mathfrak{A} \mid \operatorname{dim} \mathcal{L}\left(\Lambda_{\lambda, \mu ; \omega}^{\chi}\right)<\infty\right\} .
$$

Theorem 3.7. Let $(\lambda, \mu) \in \operatorname{Fin}_{\omega}^{\chi}$. Then there exists a $Z_{\lambda, \mu ; \omega}^{\chi} \in \mathfrak{Z}_{\omega}(\chi, \pi)$ such that $\mathfrak{H} \mathfrak{C}_{\omega}^{\chi, \pi}\left(Z_{\lambda, \mu ; \omega}^{\chi}\right)=\sum_{\nu \in \mathfrak{A}_{\pi}^{+}} \hat{\rho}^{\chi, \pi}(\nu) \breve{m}_{\nu} K_{\lambda+\nu} L_{\mu-\nu}$, where $\breve{m}_{\nu}:=\operatorname{dim} U_{-\nu}^{-} v_{\Lambda_{\lambda, \mu ; \omega}^{\chi}}$.

Proof. The claim follows from Proposition 3.5 and Lemmas 2.1 and 3.6.

Now we state the conjecture below.

Conjecture. The set $\left\{Z_{\lambda, \mu ; \omega}^{\chi} \mid(\lambda, \mu) \in \operatorname{Fin}_{\omega}^{\chi}\right\}$ must be a $\mathbb{K}$-basis of $\mathfrak{Z}_{\omega}(\chi, \pi)$.

Remark 3.8. The above conjecture fits into [18].

\section{$3.5 \mathbb{Z} / 3 \mathbb{Z}$-quantum group}

Assume that the characteristic of $\mathbb{K}$ is not 2 or 3 . Assume $l=2$. Then $I=J_{1,2}$. Assume $\mathfrak{A}=\mathfrak{A}_{\pi}$. Let $V$ be a two dimensional $\mathbb{R}$-linear space such that $\mathfrak{A} \subset V$ and $\left\{\alpha_{1}, \alpha_{2}\right\}$ is an $\mathbb{R}$-basis of $V$. Namely $\mathfrak{A}=\mathfrak{A}_{\pi}=\mathbb{Z} \alpha_{1} \oplus \mathbb{Z} \alpha_{2} \subset V=\mathbb{R} \alpha_{1} \oplus \mathbb{R} \alpha_{2}$. Let $\zeta \in \mathbb{K}$ be such that $\zeta^{2}+\zeta+1=0$. Let $q \in \mathbb{K} \backslash\{0,1, \zeta\}$.

$$
\text { Let } \chi\left(\alpha_{1}, \alpha_{1}\right)=\zeta, \chi\left(\alpha_{2}, \alpha_{2}\right)=q \text { and } \chi\left(\alpha_{1}, \alpha_{2}\right) \chi\left(\alpha_{2}, \alpha_{1}\right)=q^{-1} \text {. }
$$

Let $\beta_{1}:=\alpha_{1}, \beta_{2}:=2 \alpha_{1}+\alpha_{2}, \beta_{3}:=\alpha_{1}+\alpha_{2}$, and $\beta_{4}:=\alpha_{2}$. Then $R_{\chi}^{\pi,+}=\left\{\beta_{t} \mid t \in\right.$ $\left.J_{1,4}\right\}$. Let $\gamma_{1}:=-\beta_{4}, \gamma_{2}:=-\beta_{3}, \gamma_{3}:=-\beta_{2}, \gamma_{4}:=-\beta_{1}, \gamma_{5}=\beta_{4}, \gamma_{6}=\beta_{3}$, $\gamma_{7}=\beta_{2}$, and $\gamma_{8}=\beta_{1}$. For $t \in J_{1,8}$, let $\gamma_{t}^{\prime}:=\gamma_{s}$ with $s-t-2 \in 8 \mathbb{Z}$. For $h=\left(h_{1}, \ldots, h_{8}\right) \in \mathbb{R}_{\geq 0}^{8}$ and $t \in J_{1,8}$, let $\gamma_{t}^{(h)}:=\sum_{s=1}^{t} h_{t} \gamma_{t}$. Let $\gamma_{0}^{(h)}:=0 \in V$. Let $B_{h}:=\cup_{t=1}^{8}\left\{\gamma_{t-1}^{(h)}+u \gamma_{t} \mid 0 \leq u \leq h_{t}\right\}$, and $C_{h}:=\cap_{t=1}^{8}\left(\gamma_{t-1}^{(h)}+\mathbb{R} \gamma_{t}+\mathbb{R}_{>0} \gamma_{t}^{\prime}\right)$.

Let $\Lambda: U^{0}(\chi, \pi) \rightarrow \mathbb{K}$ be a $\mathbb{K}$-algebra homomorphism. Let $l_{i}:=\Lambda\left(K_{\alpha_{i}} L_{-\alpha_{i}}\right)$ $(i \in I)$. Assume $\operatorname{dim} \mathcal{L}(\Lambda)<\infty$. Let $\breve{m}_{\nu}:=\operatorname{dim} U_{-\nu}^{-} v_{\Lambda} . \quad\left(\nu \in \mathfrak{A}_{\pi}^{+}\right)$. By [3. Lemma 6.6] and its proof, we can see that there exists a unique $h^{\Lambda}=$ $\left(h_{1}^{\Lambda}, \ldots, h_{8}^{\Lambda}\right) \in \mathbb{Z}_{>0}^{8}$ such that $\gamma_{8}^{\left(h^{\Lambda}\right)}=0,\left\{\nu \in \mathfrak{A}_{\pi}^{+} \mid \breve{m}_{\nu}>0\right\} \subset\left(B_{h^{\Lambda}} \cup C_{h^{\Lambda}}\right) \cap \mathfrak{A}$, and $B_{h^{\Lambda}} \cap \mathfrak{A}_{\pi}^{+} \subset\left\{\nu \in \mathfrak{A}_{\pi}^{+} \mid \breve{m}_{\nu}=1\right\}$. By [3, (4.15)], we have $h_{2}^{\Lambda}, h_{4}^{\Lambda}, h_{6}^{\Lambda}, h_{8}^{\Lambda} \in J_{0,2}$ and, if $\hat{o}(q) \neq 0, h_{1}^{\Lambda}, h_{3}^{\Lambda}, h_{5}^{\Lambda}, h_{7}^{\Lambda} \in J_{0, \hat{o}(q)-1}$. Assume $\hat{o}(q)=0$. Then $\mathcal{L}(\Lambda)=\{0\}$ or there exists $(m, n) \in \mathbb{Z}_{\geq 0} \times\left(2+\mathbb{Z}_{\geq 0}\right)$ such that $l_{2}=q^{m}, l_{1}^{2} l_{2}=\left(\zeta q^{-1}\right)^{n}$, $h_{2 t}^{\Lambda}=2\left(t \in J_{1,4}\right), h_{1}^{\Lambda}=h_{5}^{\Lambda}=m$ and $h_{3}^{\Lambda}=h_{7}^{\Lambda}=n$. (See also [20, Theorem 4.1], [3, Theorem 7.8. (cK-4)].) Let $H_{1}:=\left\{q^{s} \mid s \in \mathbb{Z}\right\}$ and $H_{2}:=\left\{\left(\zeta q^{-1}\right)^{t} \mid t \in \mathbb{Z}\right\}$. Assume $\hat{o}(q) \neq 0$. If $l_{2} \in H_{1}$, then $h_{8}^{\Lambda}=h_{2}^{\Lambda}$ and $h_{6}^{\Lambda}=h_{4}^{\Lambda}$. If $l_{1}^{2} l_{2} \in H_{2}$, then 
$h_{8}^{\Lambda}=h_{6}^{\Lambda}$ and $h_{2}^{\Lambda}=h_{4}^{\Lambda}$. For example, letting $\mathbb{K}=\mathbb{C}, \xi:=\exp \left(\frac{2 \pi i}{15}\right)(\in \mathbb{C})(i$ means the imaginary unit), $\zeta:=\xi^{5}$ and $q:=\xi^{2}$, if $l_{1}=1$ and $l_{2}=q$, then $h_{1}^{\Lambda}=1$, $h_{2}^{\Lambda}=h_{8}^{\Lambda}=0, h_{3}^{\Lambda}=h_{7}^{\Lambda}=4 h_{4}^{\Lambda}=h_{6}^{\Lambda}=1$, and $h_{5}^{\Lambda}=0$.

By the above argument and Theorems 2.4 and 3.7, we can easily convince ourself that the conjecture (3.13) for almost all $\chi$ of (3.14) must be true.

\section{Appendix}

In this section, we mention a background of Theorem 2.2. Let $I=J_{1, l}$ be that of Subsections 2.1 and 2.2. Let $\mathbb{X}(I)$ be the set of all maps from $\mathbb{N}$ to $I$. Let the equivalence relation $\sim$ on the set $\mathbb{X}(I) \times \mathbb{Z}_{\geq 0}$ be such that $(f, t) \sim\left(f^{\prime}, t^{\prime}\right)$ if and only if $t=t^{\prime}$ and $f(k)=f^{\prime}(k)$ for all $k \in J_{1, t}$. Let $\mathbb{X}^{\sim}(I)$ be the quotient set $\left(\mathbb{X}(I) \times \mathbb{Z}_{\geq 0}\right) / \sim$ of $\mathbb{X}(I) \times \mathbb{Z}_{\geq 0}$ by $\sim$. Let $x_{f, t}$ be the element of $\mathbb{X}^{\sim}(I)$ such that $(f, t)$ is a representative of it. Regard $\mathbb{X}^{\sim}(I)$ as the totally ordered set $\left(\mathbb{X}^{\sim}(I), \leq\right)$ such that $x_{f, t}>x_{f^{\prime}, t^{\prime}}$ if $t \geq 1, t^{\prime} \geq 1, f(s)=f^{\prime}(s)\left(s \in J_{1, t^{\prime \prime}-1}\right.$ and $f\left(t^{\prime \prime}\right)<f^{\prime}\left(t^{\prime \prime}\right)$ for some $t^{\prime \prime} \in J_{1, t} \cap J_{1, t^{\prime}}$ or if $t<t^{\prime}$ and $f(s)=f^{\prime}(s)\left(s \in J_{1, t}\right)$. (This is the lexicographical order in the sense of [16, Subsection 1.2].) We call $x_{f, t}$ a word. We call $x_{f, 0}$ an empty word. For $(f, t) \in \mathbb{X}(I) \times \mathbb{Z}_{\geq 0}$, define $f^{+, t} \in \mathbb{X}(I)$ by $f^{+, t}(k):=f(t+k)(k \in \mathbb{N})$. We also regard $\mathbb{X}^{\sim}(I)$ as the monoid such that its unit is $x_{f, 0}$ and the multiplication is defined in the way that $x_{f, t} x_{f+, t, k}=x_{f, t+k}$. For $i \in I$, let $x_{i}:=x_{f, 1}$ for which $f$ is such that $f(1)=i$. Then $\mathbb{X}^{\sim}(I)$ can also be regarded as the free monoid generated by $x_{i}(i \in I)$.

Let $\mathbb{X}^{\sim}(I)^{\prime}:=\left\{x_{f, t} \in \mathbb{X}^{\sim}(I) \mid t \geq 1\right\}$ and $\mathbb{X}^{\sim}(I)^{\prime \prime}:=\left\{x_{f, t} \in \mathbb{X}^{\sim}(I) \mid t \geq 2\right\}$.

We say that $u=x_{f, t} \in \mathbb{X}^{\sim}(I)$ is a standard word (or a Lyndon-Shishov word) if $t \in J_{0,1}$ or if $u \in \mathbb{X}^{\sim}(I)^{\prime \prime}$ and $u>w v$ for all $v, w \in \mathbb{X}^{\sim}(I)^{\prime}$ with $v w=u$. Let $\mathbb{L} \mathbb{S}^{\sim}(I)$ be the set of all standard words. Let $\mathbb{L} \mathbb{S}^{\sim}(I)^{\prime}:=\mathbb{L} \mathbb{S}^{\sim}(I) \cap \mathbb{X}^{\sim}(I)^{\prime}$. and $\mathbb{L} \mathbb{S}^{\sim}(I)^{\prime \prime}:=\mathbb{L} \mathbb{S}^{\sim}(I) \cap \mathbb{X}^{\sim}(I)^{\prime \prime}$. It is well-known [16, Corollary1.1] that

$$
\forall u \in \mathbb{L S}^{\sim}(I)^{\prime \prime}, \exists v, \exists w \in \mathbb{L S}^{\sim}(I)^{\prime} \text { s.t. } u=v w, v>w .
$$

For $u \in \mathbb{L S}^{\sim}(I)^{\prime \prime}$, let $\dot{u}, \ddot{u} \in \mathbb{L} \mathbb{S}^{\sim}(I)^{\prime}$ be such that $u=\dot{u} \ddot{u}$ and there exists $y \in \mathbb{X}^{\sim}(I)$ with $v=\dot{u} y$ for all $v, w \in \mathbb{L}^{\sim}(I)^{\prime}$ satisfying $u=v w$ ([16, Theorem 1.1] implies $\dot{u}>\ddot{u}$ and $v>w)$. Let $\mathbb{K} \mathbb{X}^{\sim}(I)$ be the $\mathbb{K}$-linear space such that $\mathbb{X}^{\sim}(I)$ is its $\mathbb{K}$-base. Regard $\mathbb{K X}^{\sim}(I)$ as the associative $\mathbb{K}$-algebra such that $\mathbb{X}^{\sim}(I)$ is a submonoid of $\mathbb{K} \mathbb{X}^{\sim}(I)$. Then $\mathbb{K X}^{\sim}(I)$ can also be regarded as the free $\mathbb{K}$-algebra generated by $x_{i}(i \in I)$.

Let $\chi: \mathfrak{A} \times \mathfrak{A} \rightarrow \mathbb{K}^{\times}, \alpha_{i}(i \in I)$ and $\mathfrak{A}_{\pi}^{+}$be as in Subsections 2.1 and 2.2 . For $x_{f, t} \in \mathbb{X}^{\sim}(I)$, let $\theta\left(x_{f, t}\right):=\sum_{k=1}^{t} \alpha_{f(k)}\left(\in \mathfrak{A}_{\pi}^{+}\right)$. For $u=x_{f, t} \in \mathbb{L S}^{\sim}(I)$, define $[u] \in \mathbb{K} \mathbb{X}^{\sim}(I)$ by

$$
[u]:= \begin{cases}u & \text { if } t \in J_{0,1}, \\ {[\dot{u}][\ddot{u}]-\chi(\theta(\ddot{u}), \theta(\dot{u}))^{-1}[\ddot{u}][\dot{u}]} & \text { if } t \geq 2 .\end{cases}
$$

We call $[u]$ a super-letter. 
Theorem 4.1. (See [16, Theorems 1.1, 2.6 and Lemma 2.6]) (1) The set (4.1)

$$
\{1\} \cup\left\{\left[u_{1}\right]\left[u_{2}\right] \cdots\left[u_{k}\right] \mid k \in \mathbb{N}, u_{r} \in \mathbb{L} \mathbb{S}^{\sim}(I)^{\prime}\left(r \in J_{1, k}\right), u_{r} \leq u_{r+1}\left(r \in J_{1, k-1}\right)\right\}
$$

is a $\mathbb{K}$-basis of $\mathbb{K} \mathbb{X}^{\sim}(I)$. Moreover, for $v_{1}, v_{2} \in \mathbb{L} \mathbb{S}^{\sim}(I)^{\prime}$ with $v_{1}>v_{2},\left[v_{1}\right]\left[v_{2}\right]$ is a linear combination of elements $\left[u_{1}\right]^{n_{1}}\left[u_{2}\right]^{n_{2}} \cdots\left[u_{k}\right]^{n_{k}}$ in (4.1) with $v_{2} \leq u_{1}$, $v_{1} \leq u_{k}$, and $\theta\left(u_{1}^{n_{1}} u_{2}^{n_{2}} \cdots u_{k}^{n_{k}}\right)=\theta\left(v_{1}\right)+\theta\left(v_{2}\right)$.

(2) The same claim as that of (1) with the monomials $u_{1} u_{2} \cdots u_{k}$ in place of $\left[u_{1}\right]\left[u_{2}\right] \cdots\left[u_{k}\right]$ is true.

Let $\dot{U}^{+, b}$ be a Hopf $\mathbb{K}$-algebra generated by $\dot{K}_{\lambda}(\lambda \in \mathfrak{A})$, and $\dot{E}_{i}(i \in I)$ satisfying the same equations as those of (2.4) and (2.5) with $K_{\lambda}$ and $E_{i}$ in place of $K_{\lambda}$ and $E_{i}$ respectively. Let $U^{+, b, 0}$ (resp. $U^{+}$) be the $\mathbb{K}$-subalgebra of $U^{+, b}$ generated by $K_{\lambda}(\lambda \in \mathfrak{A})\left(\right.$ resp. $\left.E_{i}(i \in I)\right)$. Note $\dot{U}^{+, b}=\operatorname{Span}_{\mathbb{K}}\left(\dot{U}^{+} \dot{U}^{+, b, 0}\right)$. Let $\dot{G}:=\left\{\dot{K}_{\lambda} \mid \lambda \in \mathfrak{A}\right\}$. Assume that $\dot{G}$ is a $\mathbb{K}$-basis of $\dot{U}^{+, b, 0}$, and that $\dot{K}_{\lambda} \neq \dot{K}_{\mu}$ for $\lambda \neq \mu$. Assume that $\dot{G}=\left\{g \in \dot{U}^{+, b} \mid \Delta(g)=g \otimes g\right\}$. (Such Hopf algebra is called a Character Hopf algebra, see [16, Defnnition 1.11].) Let $\Gamma=(\Gamma, \preceq)$ be a well-ordered additive (commutative) monoid, see [14 for this terminology.

Example 4.2. Let $n \in \mathbb{N}$. Define the well-order $\preceq$ on $\mathbb{Z}_{\geq 0}^{n}$ in the way that for $k=\left(k_{1}, \ldots, k_{n}\right), r=\left(r_{1}, \ldots, r_{n}\right) \in \mathbb{Z}_{\geq 0}^{n}$, we have $k \prec r$ if and only if $\sum_{t=1}^{n} k_{t}<\sum_{t=1}^{n} r_{t}$ or there exists $b \in J_{1, n}$ with $k_{b}<r_{b}$ and $k_{b^{\prime}}=r_{b^{\prime}}$ for all $b^{\prime} \in J_{1, b-1}$. Then $\left(\mathbb{Z}_{\geq 0}^{n}, \preceq\right)$ is a well-ordered additive monoid. Note that $\mathfrak{A}_{\pi}^{+}$is a well-ordered additive monoid in a natural sense.

Let $D: \mathbb{X}^{\sim}(I) \rightarrow \Gamma$ be a monoid homomorphism such that $0 \notin D\left(\mathbb{X}^{\sim}(I)^{\prime}\right)$. Also, let $D\left(\left[u_{1}\right]\left[u_{2}\right] \cdots\left[u_{k}\right]\right)$ mean $D\left(u_{1} u_{2} \cdots u_{k}\right)$. Define the $\mathbb{K}$-algebra epimorphism $\Phi: \mathbb{K X}^{\sim}(I) \rightarrow U^{+}$by $\Phi\left(x_{i}\right):=E_{i}(i \in I)$. For the element $X=$ $\left[u_{1}\right]\left[u_{2}\right] \cdots\left[u_{k}\right]$ in (4.1), let $M(X):=u_{k}$. Let $\mathbb{B}$ be the set in (4.1). For $u \in$ $\mathbb{L S}^{\sim}(I)^{\prime}$, we call $[u]$ hard if $\Phi([u]) \notin \operatorname{Span}_{\mathbb{K}}(\{\Phi(X) \mid X \in \mathbb{B}, D(X)=D(u), M(X)<$ $u\} \cup\{g \Phi(Y) \mid g \in G, Y \in \mathbb{B}, D(Y)<D(u)\}$ ), see [16, Definition 2.6 (see also Lemma 2.6)]. For a hard super-letter $[u]$, let $H([u]):=\left\{k \in \mathbb{N} \mid \Phi\left([u]^{k}\right) \in\right.$ $\operatorname{Span}_{\mathbb{K}}\left(\left\{\Phi(X) \mid X \in \mathbb{B}, D(X)=D\left(u^{k}\right), M(X)<u\right\} \cup\{g \Phi(Y) \mid g \in \dot{G}, Y \in \mathbb{B}, D(Y)<\right.$ $\left.\left.\left.D\left(u^{k}\right)\right\}\right)\right\}$. If $H([u]) \neq \emptyset$, let $h([u]):=\min H([u])$. Otherwise, let $h([u]):=\infty$. Clearly $h([u]) \geq 2$.

Theorem 4.3. ([16, Defintion 2.7, Theorems 2.2 and 2.4 (see also Lemma 2.6)]) (1) Let $h:=h([u])$. If $h<\infty$, then $\chi(\theta(u), \theta(u))$ is a primitive $t$-th root of unity for some $t \in \mathbb{N}$, and $h=t p^{r}$ for some $r \in \mathbb{Z}_{\geq 0}$, where $p:=\operatorname{Char}(\mathbb{K})(\in$ $\{0\} \cup(\mathbb{N} \backslash\{1\})$.

(2) Let $\mathbb{H}$ be the set of hard super-letters. Then the elements

$$
g \Phi\left(\left[u_{1}\right]^{n_{1}}\left[u_{2}\right]^{n_{2}} \cdots\left[u_{k}\right]^{n_{k}}\right) \quad\left(g \in \dot{G},\left[u_{i}\right] \in \mathbb{H}, n_{i}<h\left(\left[u_{i}\right]\right), u_{1}<\cdots<u_{k}\right)
$$

form a $\mathbb{K}$-base of $\dot{U}^{+, b}$.

(3) The same claim as that of (2) with the monomials $u_{1}^{n_{1}} u_{2}^{n_{2}} \cdots u_{k}^{n_{k}}$ in place of $\left[u_{1}\right]^{n_{1}}\left[u_{2}\right]^{n_{2}} \cdots\left[u_{k}\right]^{n_{k}}$ is true. 
Assume that we have the $\mathbb{K}$-linear isomorphism $\varsigma^{\prime}: U^{+} \otimes U^{+, b, 0} \rightarrow U^{+, b}$ defined by $\varsigma^{\prime}(X \otimes Z):=X Z$. By Theorem 4.3 , we can easily see that

$$
\begin{aligned}
& \left\{\Phi\left(\left[u_{1}\right]^{n_{1}}\left[u_{2}\right]^{n_{2}} \cdots\left[u_{k}\right]^{n_{k}}\right) \mid\left[u_{i}\right] \in \mathbb{H}, n_{i}<h\left(\left[u_{i}\right]\right), u_{1}<\cdots<u_{k}\right\} \\
& \text { is a } \mathbb{K} \text {-basis of } \dot{U}^{+} .
\end{aligned}
$$

(The claim of $\left[u_{1}\right]^{n_{1}}\left[u_{2}\right]^{n_{2}} \cdots\left[u_{k}\right]^{n_{k}}$ can be replaced by $u_{1}^{n_{1}} u_{2}^{n_{2}} \cdots u_{k}^{n_{k}}$.) Let $\mathbb{H}:=$ $\mathbb{H} \cup\left\{[u]^{\hat{o}(\chi(\theta(u), \theta(u))) p^{s}} \mid[u] \in \mathbb{H}, s \in \mathbb{Z}_{\geq 0}, 0<\hat{o}(\chi(\theta(u), \theta(u))) p^{s}<h([u])\right\}$, where $p:=\operatorname{Char}(\mathbb{K})$. Define the total order $<$ on $\mathbb{H}$ in the way that $[u]^{x}>[v]^{y}$ if and only if $u>v$ or $u=v$ and $x<y$. Define the map $h: \mathbb{H} \rightarrow(\mathbb{N} \backslash\{1\}) \cup\{\infty\}$ as follows. If $\hat{o}(\chi(\theta(u), \theta(u)))=0$, let $\hat{h}([u]):=\infty(=h([u]))$. Otherwise, let $\hat{h}\left([u]^{x}\right):=\hat{o}\left(\chi\left(\theta\left(u^{x}\right), \theta\left(u^{x}\right)\right)\right)$. Then the same claim as (4.2) with $\mathbb{H}$ and $\dot{h}$ in place of $\mathbb{H}$ and $h$ is true.

Assume that we have a direct sum $U^{+}=\oplus_{\lambda \in \mathfrak{A}_{\pi}^{+}}{U_{\lambda}^{+}}_{\lambda}$ as a $\mathbb{K}$-linear space with $1 \in \dot{U}_{0}^{+}, \dot{E}_{i} \in \dot{U}_{\alpha_{i}}^{+}$and $\dot{U}_{\lambda}^{+} \dot{U}_{\mu}^{+} \subset \dot{U}_{\lambda+\mu}^{+}$. Let $\dot{R}_{\chi}^{+}:=\left\{\theta\left(u^{x}\right) \mid[u]^{x} \in \mathbb{H}\right\}$. Define the $\operatorname{map} \dot{\varphi}_{\chi}^{+}: \hat{R}_{\chi}^{+} \rightarrow \mathbb{N}$ by $\dot{\varphi}_{\chi}^{+}(\beta):=\left|\left\{[u]^{x} \in \mathbb{H} \mid \theta\left(u^{x}\right)=\beta\right\}\right|$. Then the pair $\left(\dot{R}_{\chi}^{+}, \dot{\varphi}_{\chi}^{+}\right)$ is independent from choice of $D$ (and $\Gamma$ ). Recall $U^{+, b}$ from the sentence below (2.5). Identify $\dot{U}^{+, b}$ with $U^{+, b}$ in a natural way. Then $\left(\dot{R}_{\chi}^{+}, \dot{\varphi}_{\chi}^{+}\right)$can be identified with $\left(R_{\chi}^{\pi,+}, \varphi_{\chi}^{\pi,+}\right)$ of Theorem [2.2. Let $[u]^{x} \in \mathbb{H}, \alpha:=\theta\left([u]^{x}\right), t \in J_{1, \hat{\varphi}_{\chi}^{+}(\alpha)}$ and $d:=(\alpha, t)$. Then we have $y(d) \in J_{0, \hat{h}\left([u]^{x}\right)-1}$, where $y$ is the one of Theorem 2.2 .

\section{References}

[1] I. Angiono and H. Yamane, The R-matrix of quantum doubles of Nichols algebras of diagonal type, J. Math. Phys. 56, (2015) 021702.

[2] , Bruhat order and nil-Hecke algebras for Weyl groupoids, J. Algebra Appl. 17 (2018), no. 9, 1850166.

[3] S. Azam, H. Yamane and M. Yousofzadeh, Classification of Finite Dimensional Irreducible Representations of Generalized Quantum Groups via Weyl Groupoids, preprint, Publ. Res. Inst. Math. Sci. 51 (2015), no. 1, 59-130.

[4] P. Batra and H. Yamane, Skew centers of rank-one generalized quantum groups, Toyama Mathematical Journal, Volume 37 (2015), 189-202.

[5] Centers of generalized quantum groups, J. Pure Appl. Algebra 222 (2018), no. 5, 1203-1241.

[6] M. Cuntz and I. Heckenberger, Finite Weyl groupoids, J. Reine Angew. Math. 702 (2015), 77-108.

[7] V.G. Drinfel'd, Quantum groups, Proceedings of the International Congress of Mathematicians, Vol. 1, 2 (Berkeley, Calif., 1986), 798-820, Amer. Math. Soc., Providence, RI, 1987. 
[8] I. Heckenberger, The Weyl groupoid of a Nichols algebra of diagonal type, Invent, Math. 164 (2006) no, 1, 175-188.

[9] Nichols algebras, ECNU Shanghai, July 2008 http://www.mathematik.uni-marburg.de/ heckenberger/na.pdf

[10] Classification of arithmetic root systems, Adv. Math. 220 (2009), no. $1,59-124$.

[11] I. Heckenberger and H. Yamane, A generalization of Coxeter groups, root systems, and Matsumoto's theorem, Math. Z. 259 (2008), 255-276.

[12] - Drinfel'd doubles and Shapovalov determinants, Revista de la Union Matematica Argentina vol. 51-2 (2010), 107-146.

[13] N. Jing, K. Misra, H. Yamane, Kostant-Lusztig $\mathbb{A}$-bases of multiparameter quantum groups, Representations of Lie algebras, quantum groups and related topics, 149-164, Contemp. Math., 713, Amer. Math. Soc., Providence, RI, 2018.

[14] Y. Kobayashi, Well-ordered monoids -two numerical functions-, Surikaiseki Kenkyusho Kokyuroku, RIMS, vol. $1366 \quad$ (2004), 111-120. https://repository.kulib.kyotou.ac.jp/dspace/bitstream/2433/25368/1/1366-13.pdf

[15] V. Kharchenko, A quantum analogue of the Poincaré-Birkhoff-Witt theorem, Algebra and Logic 38 (1999), no. 4, 259-276.

[16] _ Quantum Lie theory. A multilinear approach. Lecture Notes in Mathematics, 2150. Springer, Cham, 2015. xiii+302 pp. ISBN: 978-3-31922703-0; 978-3-319-22704-7

[17] G. Lusztig, Introduction to quantum groups, Birkhäuser Boston, Inc., Boston, MA, 1993.

[18] A. Sergeev and A. Veselov, Grothendieck rings of basic classical Lie superalgebras, Ann.

[19] T. Tanisaki, Killing forms, Harish-Chandra isomorphisms, and universal $R$ matrices for quantum algebras, Infinite Analysis (Proceedings of RIMS symposium, Kyoto, Japan, June-August 1991) ed, by A. Tsuchiya, T. Eguchi and M. Jimbo, World scientific Singapore, 1992, 941-961.

[20] H. Yamane, Representations of a $\mathbb{Z} / 3 \mathbb{Z}$-quantum group, Publ. RIMS, Kyoto Univ., 43 (2007), 75-93. 
Punita Batra, Harish-Chandra Research Institute, Chhatnag Road, Jhunsi, Allahabad 211 019, India,

E-mail: batra@hri.res.in

Hiroyuki Yamane, Department of Mathematics, Faculty of Science, University of Toyama, 3190 Gofuku, Toyama-shi, Toyama 930-8555, Japan, E-mail: hiroyuki@sci.u-toyama.ac.jp 